\title{
El consumo digno y los negocios de app de taxi (caso Uber)'
}

\section{Dignified consumption and taxi app business (Uber case)}

\author{
Carlos Eduardo Armas Morales ${ }^{2}$
}

\section{RESUMEN}

El presente artículo, dentro del marco actual de la denominada economía colaborativa, evalúa si las app de taxis (Uber) que ofertan servicios en el Perú (Lima), lo hacen concordando con los principios mínimos del consumo digno, es decir si brindan un buen servicio de transporte de taxi, teniendo en cuenta que en Europa estos servicios han sido clasificados dentro del transporte público de taxis (Sentencia del Tribunal Europeo el año 2017). Y de otro lado, existe el debate en Latinoamérica, incluido el Perú de regular estos servicios (se presentaron dos proyectos de ley en

\begin{abstract}
1 Promover la investigación en el Pre grado en el curso de derecho económico de la Facultad de Ciencias Económicas, motiva que estos alumnos en forma grupal realicen algunas investigaciones sobre temáticas del curso que finaliza con la entrega de un ensayo de artículo científico con sus respectivos anexos si fuera necesario, lo cual el docente selecciona de acuerdo a ciertos parámetros para su difusión, en este caso el anexo de un artículo de grupo de alumnos del curso de derecho económico 206-N, 2017II , nos trajo los resultados de una encuesta sobre la problemáticas de los app de taxis (caso UBER) en el Perú y en el mundo sobre su regulación. De otro lado siendo perentorio igualmente difundir algunas técnicas aprendidas en el seminario de investigación cualitativa como la triangulación de datos e información, dictada por la Dra. María Da Conceicao Ferreira Monteiro profesora de la Universidad de ÉVORA (Portugal), aspectos que nos ha motivado escribir este artículo en el afán de reflexionar sobre el consumo-servicio digno del transporte de taxi con aplicaciones digitales que se ve empañado por actos delincuenciales debido a la inseguridad públicas en Lima y otros lugares del país.

2 Ingeniero Industrial (UNI) - Abogado (UNSMM), con estudios de Maestría en Impacto territoriales y ambientales de la Globalización (Becario de la Universidad Internacional de Andalucía - España), Magister y Doctor en Derecho UNMSM. Profesor Asociado en la Facultad de Ciencias Económicas. Investigador titular del grupo de Investigación INCIRENA. Pabellón de la Facultad de Ciencias Económicas, calle Germán Amezága 375, Lima 1 -Perú, Teléfono 990115042.

Correo electrónico: carmasm@unmsm.edu.pe
\end{abstract}

(C) Los autores. Este artículo es publicado por Pensamiento Crítico de la Facultad de Ciencias Económicas, Universidad Nacional Mayor de San Marcos. Este es un artículo de acceso abierto, distribuido bajo los términos de la licencia Creative Commons Atribucion - No Comercia_Compartir Igual 4.0 Internacional. (http:// creativecommons.org/licenses/by-nc-sa/4.0/) que permite el uso no comercial, distribución y reproducción en cualquier medio, siempre que la obra original sea debidamente citada. 
el 2017) , lo que nos convoca a una revisión teórica dentro del marco del derecho económico y contractual peruano, evaluando siestas cumple con el consumidor: usuario-cliente, con la garantía de una seguridad adecuada al resguardo de su vida, salude integridad psíquica física, es decir si el servicio es seguro para el usuariocliente, en el sentido de tener un trato digno en su consumo y dado los hechos delictivos (robos, intentos de violación y secuestros etc.) que han ocurrido y ocurren en Lima en la prestación de este servicio, no obstante creemos que este estudio reflejaría lo que ocurre en otros lugares del Perú.

Palabras clave: Economía colaborativa; usuario colaborativo; transporte de taxi.

\section{ABSTRACT}

This article, within the current framework of the so-called collaborative economy, evaluates whether the taxi app (Uber) that offers services in Peru (Lima), do so in accordance with the minimum principles of decent consumption, that is, if they provide a good taxi transport service, taking into account that in Europe these services have been classified within the public transport of taxis (Judgment of the European Court in 2017). And on the other hand, there is a debate in Latin America, including Peru to regulate these services (two bills were submitted in 2017), which calls for a theoretical review within the framework of Peruvian economic and contractual law, evaluating whether these meets the consumer: user-client, with the guarantee of adequate security to safeguard their life, health and physical psychic integrity, ie if the service is safe for the user-client, in the sense of having a decent treatment in its consumption and given the criminal acts (robberies, attempted rape and kidnappings etc.) that have occurred and occur in Lima in the provision of this service, nevertheless we believe that this study would reflect what is happening in other parts of Peru.

Keywords: Collaborative economy; collaborative user; taxi transport.

\section{Introducción}

La Constitución de la república peruana establece en su artículo 1, que la defensa de la persona humana y el respeto a su dignidad son el fin supremo de la sociedad y del Estado, ahora bien centrándonos en la noción del vocablo dignidad de la frase "respeto a su dignidad", nos lleva ineludiblemente al termino digno, que correlativamente el Diccionario de la Real Academia Española (RAE), lo define -entre otras acepciones- como : merecedor de algo// correspondiente, proporcionado al mérito y condición de alguien o algo. RAE (2014, p.799); que nos invita a discernir ¿Qué es el trato digno de un ser humano en la sociedad?, que trasladado a una relación social significaría tratar con honor, respeto o consideración que 
le corresponde a una persona cuando se respeta su dignidad, y si bien nuestra constitución lo alude así en su primer artículo, el concepto de dignidad asume una retahíla de interpretaciones que no se agotan en algún concepto instrumental de los autores si no que reflejan mucho más, no obstante alguno expertos como Martínez, nos da una acepción, que es permisible y comprensible en su sentir humanista, tal como el de "reconocer la necesidad de tratar a cada uno de los seres como lo que es..., pues lo verdaderamente importante para el hombre es ser tratado como lo que es, de acuerdo con sus atributos y características" Agregando que: es necesario reflexionar y establecer consensos sociales que se traduzcan en pautas de conducta e incluso en regulaciones jurídicas, que nos permitan clarificar la manera en que la concepción de la dignidad humana debe traducirse en la práctica en nuestra vida cotidiana, cada vez más compleja. Martínez (2012, p. 1-16)

Al amparo de lo dicho, y relacionándolo con el consumo, podríamos decir que consumo digno, ventilada dentro de una sociedad decente, es aquel honor y respeto con que se le debe tratar a las personas, en cuanto le corresponda asumir el rol de consumidor, que ejercitando el consumo de un bien o servicio en un mercado, merece el trato de humanidad o de humano por la otredad, respetando por lo mismo los correspondientes derechos que posee y merece el ciudadano consumidor.

Desde la óptica del consumo de transporte público de taxis de aplicaciones digitales, somos conscientes por lo menos en el Perú (Lima), de diversas situaciones ilegitimas e ilegales que han sufrido los consumidores de este tipo de transporte, que han solicitado estos servicios y en especial de la empresa Uber, cuyas evidencias son expuestos en reportes periodístico, que dan cuenta de estos accidentes e incidente reales así como el comportamiento empresarial de esta empresa en relación a su carencia de humanidad y dignidad hacia el homo consumens, situación que se ven complementadas coadyuvados por la técnica de triangulación de fuentes, con las las opiniones de algunos usuarios de este servicio, que nos indican su preocupación por la seguridad (cuadro No 1).

De otro lado la reciente resolución ECLI:EU:C:2917:981 del Tribunal de Justicia de Luxemburgo (Sentencia del Tribunal de Justicia del 20 de diciembre de 2107-caso Asociación Profesional Elite Taxi Y 
UberSystemsSpain, S. L.) en adelante Tribunal de Justicia de Luxemburgo ha considerado a Uber clasificada dentro de los servicios de transporte de taxis, lo que posibilita para que en nuestros países Latinoamericanos y concretamente nuestro país se le regule así, de tal manera que el usuario pasajero, de alguna forma, en caso de accidentes o incidentes delincuenciales pueda exigir el cumplimiento de responsabilidades derivadas de tal condición, sea esta, en forma individual o solidaria contra esta empresa, lo que conllevaría de otro lado, enervar los contratos de Uber que suscribe con sus usuarios (conductor y cliente), el cual en forma unilateral y supuestamente con vigencia en territorio peruano impone su contrato; alentado seguramente por los principios de libertad empresarial, iniciativa y autonomía privada que rige nuestro mercado peruano, empero autoproclamándose sin responsabilidad legal ante la eventual circunstancia de incidentes y o accidente que sufra el cliente, posición jurídica que es contraria a la legislación contractual peruana, en el sentido que si bien el Estado Peruano, a través de nuestra carta magna y otras leyes, reconoce que la inversión nacional y o extranjera actúan en el mercado en igualdad de condiciones y asimismo, reconociendo la libertad empresarial y contractual, no obstante un contrato no es una fuente de libre albedrio que colinde con un libertinaje a ultranza, pues conlleva limitaciones de conformidad con el Estado de derecho, es decir el respeto a las leyes públicas para que tenga valor jurídico; es decir debe estar arreglado a la leyes peruanas, aspectos jurídicos que ya se estipulaba en el derecho romano a través del brocárdico latino : "Libertas estnaturalis facultas eiusquodcuiquefácere, nisi si quid vi aut iure prrohibetur", es decir la libertad es la facultad natural de hacer cada uno lo que le plazca, sin embargo está limitado por la prohibición de la fuerza de la ley, como es el caso del contrato de Uber, el que contiene cláusulas eximentes, de exoneración y o de irresponsabilidad legal que contravienen a la ley y la constitución peruana, aspectos que detallaremos más adelante.

El trabajo en un primer acápite,se ocupa de la inserción de este tipo de plataformas en los mercado del mundo y sus impactos causados en función de irrumpir la quietud de las clásicos servicios basados en la presencia humana y sus relaciones materiales que desde luego gracias a la innovación tecnológica vislumbran condiciones sociales y culturales de nuevo cuño, "lo cual desemboca en un cambio del sistema económico donde aparece la economía colaborativa que propone un cambio profundo 
de valores con respecto al consumo, la propiedad y el estatus social" Diez (2015, p.8).

En un segundo acápite se evalúa las relaciones contractuales impuestos por la empresa Uberen forma unilateral y que deja en estado de indefensión a los clientes usuarios de transporte (consumidor). Y en un tercer acápite hacemos un evaluación a la luz de la legislación peruana vigente sobre del consumo digno del transporte de taxi con aplicación (Uber) y el rol del Estado.

\section{Economia colaborativa, empresas de taxis APP y el servicio de taxis}

Si bien a estas empresas con aplicaciones por plataformas digitales, se les publicita como integrantes de la denominada economía colaborativa, nosotros creemos que ello es solo un membrete "marketero", pues para nadie es oculto, que algunas de estas empresas integrantes de dicha "economía" tienen ingresos y utilidades que superan a grandes empresas trasnacionales clásicas, así "se estima que Uber logró facturar US $\$ 10.000$ millones en el último año, como su tarifa es de $20 \%$ sobre lo facturado, los ingresos netos de la compañía alcanzarían los US \$2.000 millones; es decir, un crecimiento de $100 \%$ frente a sus ingresos estimados de 2013". Asimismo agrega esta misma fuente en relación a la valorizaciónde Uber y otras :

Uber... ha logrado una valorización superior a los US $\$ 40.000$ millones; Waze, la App de movilidad más reconocida del mundo, fue adquirida por Google en una transacción de más de US $\$ 1.000$ millones; Airbnb, que permite obtener hospedajes particulares en casi todo el planeta, acaba de ser valorada en US $\$ 13.000$ millones, y Spotify, la gran comercializadora de música en streaming, tiene 15 millones de usuarios pagando en todo el mundo. (hppt://www.dinero.com/edicion-impresa/caratula/ articulo/aplicaciones-mas-usadas-mundo-2015/208936)

Lo que nos permite advertir que la gran mayoría de estas supuestas empresas de colaboración, así como tienen grandes ingresos poseen costos y gastos inherentes a gestionar una serie de flujos económicos empresariales derivados de la comercialización, publicidad, gestión de la venta de servicios, uso de plataforma etc. Coligando que estamos ante la presencia de un negocio común y corriente que utiliza innovaciones 
tecnológicas, es decir, sin negar su novedad y el uso de la alta tecnología, incursionan como cualquier otra empresa en los mercados clásicosde empresas de taxi en las ciudades del mundo. De otro lado, en afán de escudriñar la naturaleza comercial de estas empresas, otros encuentra contradicciones en la definición e integración de varios de estos servicios dentro del rubro de economía colaborativa, al preguntarse y luego responderse:"¿Pueden equipararse prácticas de trabajar como conductor de Uber, la de prestar gratuitamente el sofá a través de Couchsurfing, o de compartir conexión mediante Wife Net ET? ...definiciones y clasificaciones... poco robustas metodológicamente y, por ello están condenadas a ser poco útiles o nada útiles" Lain (2017, pp. 107-114). Ante estas contradicciones, de haber servicios por colaboración o sea gratuitos y otros pagados, no cabe agrupar a estas empresas en el rubro de economía colaborativa, pues en el caso de Uber, esta presta servicios con un manejo empresarial rentista. Es decir no estamos ante una colaboración gratuita o de un altruismo colaborativo, si no ante un servicio de transporte que "terceriza" algunos procesos, que equivale a negociar, tal como la conducción (usuario conductor), y de otro lado, tampoco es un simple dador de acceso o perteneciente a la denominada sociedad de la información (una simple plataforma digital de intermediación) si no una empresa de prestación de servicios de taxis; conclusión a la que ha llegado el Tribunal de Justicia de Luxemburgo en la Resolución contenida en el documento ECLI:EU:C:2017: 981.A saber:

Deben interpretarse en el sentido de que ha de considerarse que un servicio de intermediación..., que tiene por objeto conectar, mediante una aplicación para teléfonos inteligentes, a cambio de una remuneración, a conductores no profesionales que utilizan su propio vehículos con personas que deseen efectuar un desplazamiento urbano, esta indisociablemente vinculado a un servicio de transporte y, por lo tanto, ha de calificarse este servicio en el ámbito de transporte...

A nuestro parecer, resolvió en forma acertada, ya que Uber no es una simple dadora de acceso, sino estaría clasificada como un sistema empresarial de prestación de servicio de taxis.

En tal sentido lo más importante de la sentencia, es que indica taxativamente que su clasificación corresponde a prestar "servicios en el ámbito de transporte" o lo que equivale a una empresa de taxis, desechando que pueda responder a la clasificación de "servicios de 
la sociedad de la información". Lo cual conlleva, si lo retrotraemos al mercado y legislación peruana, que dicho servicio deben adecuarse a la normativa de consumo e igualmente al de trabajo; pues en relación a esto, expresa Sholz(2016, p.9) "no podemos hablar sobre plataformas de trabajo, sin antes reconocer que dependen vidas humanas explotadas a lo largo de sus cadenas de suministro globales",reflexión que interpretamos, en el sentido que el autor opta, de un lado, por la valoración de la vida, sugiriendo del otro, responsabilidades ante el trabajo del trabajador y el servicio prestado al consumidor; responsabilidades que Uber trata de eludir, tal como se explica más adelante.

En cuanto al modelo de negocios, el proyecto de ley colombiano del 2016, presentada por congresista Restrepo, indica que "corresponde al modelo económico donde se le provee un servicio a un cliente por medio de una aplicación móvil o plataforma tecnológica, la cual actúa como intermediaria entre el usuario y la persona que suministra tales servicios" Restrepo (2016, p1), y tal como lo refiere en una artículo Armas (2016, p 9-25), son plataformas digitales diseñadas para interconectar con el uso de aplicaciones a pasajeros con conductores- taxistas, ambos, registrados en la red de estas empresas como es el caso de Uber, que conectados a una aplicación pueden utilizar terminales fijos o móviles como i-phone, o páginas web, pero no solo ello, dependiendo de la tecnología, puede ayudar a integrar opciones de transporte existentes en una misma experiencia tal como buscar la ruta más rápida utilizando la combinación más eficiente de viajes compartidos y transporte público, basándose en los patrones de tráfico de la localidad.

Desde otra mirada, estas empresas utilizan nuevos modelos de negocios, para insertarse en la actividad económica de los mercados de taxis, los que sin tener bienes físicos como "automóviles" en propiedad, se interconectan a través de las tecnologías internet con los que tienen dicho bien para prestar servicios de taxis a otros que solicitan este servicio; ambos sujetos a un contrato de acceso a la plataforma; denominándose contratista al : usuario- transportista (conductor); y usuario-cliente, al consumidor a quien se le brindará el servicio de transporte, firmando ambos el mismo tipo de contrato, salva diferencias por naturaleza de su función y posición contractual. Siendo más, ambos están registrados en la plataforma de estas empresas. 
De tal manera que, a la luz de la resolución ECLI:EU:C:2917:981 o sentencia de Tribunal de Justicia Luxemburgo y algunas propuestas de regulación de los países donde se incluye el Perú (En Perú se presentaron los Proyecto de Ley No. 2218/2017 y la No. 1505/2016), consideran a estos servicios app "tercerizado" como un todo sistémico que debe estar sujeto a la normativa legal, por lo tanto corresponde y siendo pertinente, revisar y reflexionar con el instrumental legal: sea doctrina, legislación o jurisprudencia, los términos que difunden estas plataformas para sus usuarios: conductores y clientes, asumiendo que estamos ante un trabajo dependiente (contrato de trabajo) del usuario conductor en relación a la plataforma y descartando el trabajo autónomo o por cuenta propia que en el Perú se denomina locación de servicios.

Mas, desde otro ángulo, siendo el temas central de nuestras reflexiones, evaluar si el consumo del servicio que presta Uber es un producto adecuado, es decir un servicio de transporte público que resguarde la integridad psíquica física y la vida del usuario o en otro sentido: un servicio seguro para el consumidor, teniendo en cuenta que en el caso del país vecino Chile, estos "ya superan mil 250 mil usuarios y 52 mil socios conductores inscritos. Estas cifras hacen que una de sus principales preocupaciones sea la seguridad" Lago (2016 pp. 16-20). En el caso del Perú, la ciudad de Lima, la seguridad pública es una de las problemáticas pendiente del gobierno. Ello nos obliga a realizar una pequeña muestra no estadística sobre el tema de la seguridad de los taxis app en Lima, para solventar el artículo y cuya información lo emplearemos para triangularlo con otras informaciones concretas y reales de inseguridad (ataques delincuenciales), que han sido publicitados en los diarios de la capital y que desde luego solventa nuestras apreciaciones.

Al respecto la definición de triangulación de datos de UweFlick (2014, p.67), refiere:

la combinación de diferentes clases de datos sobre el fondo de las perspectivas teóricas que se aplican a ellos. Estas perspectivas se deben tratar y aplicar en la medida de lo posible en pie de igualdad y siguiendo por igual los dictados de la lógica. Al mismo tiempo, la triangulación (de diferentes métodos o tipo de datos), debe permitir un excedente importante de conocimiento.

Así de acuerdo a un total de 31 personas que contestaron una encuesta en 14 distritos (Gráfico No. 1), para solventar y hacer más asequible 
este estudio, se tuvo el siguiente resultado: 10 personas no usaban este servicio y 21 si hacían el servicio de taxis en esta modalidad (64.5\%); en cuanto a la frecuencia de utilización de este grupo, el 77\% usaba de vez en cuando, es decir hay un regular uso, si bien no intensivo como en otras latitudes, hay uso en Lima. Siendo lo más importante conocer la seguridad, se preguntó (pregunta cerrada) ante varias alternativas, el por qué los usa, el $42.9 \%$ contesto porque es más seguro y el $60.9 \%$ lo consideraban bueno, siendo más, el $21.7 \%$ indico excelente; pero cuando recurrimos a la pregunta abierta sobre su opinión general sobre el servicio de taxis de Uber, de los 21 encuestados, 17 se referían a la seguridad y los riesgo de este tipo de taxis e igualmente diagnosticaban que era necesario un mejorar el control.

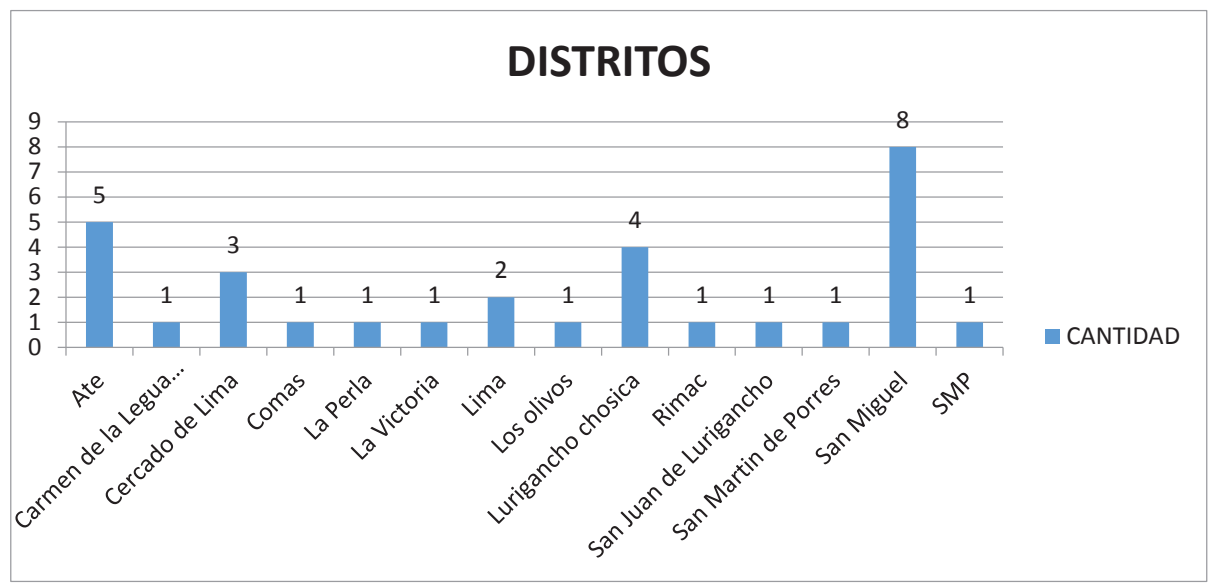

Grafico No. 1. Muestra de encuesta (personas que usan servicio de taxis app)

Fuente propia

Matizado estos datos de la muestra, con los sucesos policiales difundidos en diarios y publicitados en internet, se corroboran que si bien el usuario pasajero en Lima usa este servicio y considera algunas ventajas y otros buen servicio, creemos que la prestación de este servicio por Uber tiene brechas abiertas que los aleja de las prerrogativas de un consumo digno, en primer lugar por los sucesos negativos (robos intentos de secuestro violación) que acontecen en el mercado limeño de taxis de Uber, y desde luego, la no atención adecuada al pasajero víctima, pues 
si bien estos sucesos son mínimos, ello no es óbice para ignorarlos, pues atentaron de alguna manera contra el consumo digno de las personas ; y en segundo lugar por eludir responsabilidades en relación a estos servicios (relatados por algunos clientes), teniendo en cuenta las diversas exoneraciones, limitaciones y renuncias a su favor prescritas en el contrato que circula por la red del año 2017 para el Perú; modelo con el cual esta empresa suscribe sus contratos con los usuarios: conductor y pasajero.

Ahora bien triangulando datos e información de estas dos fuentes (encuestas y difusión de sucesos): Las respuestas de la pregunta abierta a las 21 personas encuestadas y los tres casos puntuales que hemos revisados de la información periodística se han tenido resultados convergentes, cuyas consideraciones los hemos plasmamos el cuadro No.1.

\section{Terminos y condiciones del contrato que suscribe Uber con sus usuarios}

Es un contrato de servicios que posee clausulas predispuestas a las que se sujetan los usuarios, sean estos conductores o clientes (pasajeros), es decir pertenece al contrato denominado de adhesión; lo tomas o lo dejas, en la que Uber impone sus condiciones en forma unilateral para supuestamente dar acceso o un servicio de valor añadido, asumiendo que pertenece a la sociedad de la información y desde luego sustrayéndose de ser una empresa de taxis y por lo tanto no es responsable de ningún evento, incidente y accidente que ocurra tanto al usuario conductor como al cliente.

Al respecto Uber define y fija en este contrato aludido, su posición de empresa dadora de acceso a sus usuarios a través de una plataforma acceso a la red, indicando (2. Los servicios):

Los Servicios constituyen una plataforma de tecnología que permite a los usuarios de aplicaciones móviles de Uber o páginas web proporcionadas como parte de los Servicios (cada una, una "Aplicación") organizar y planear el transporte y/o servicios de logística con terceros proveedores independientes de dichos servicios, incluidos terceros transportistas independientes y terceros proveedores logísticos independientes, conforme a un acuerdo con Uber o algunos afiliados de Uber ("Terceros proveedores").

En este mismo acápite establece en forma taxativa y en mayúsculas: 
USTED RECONOCE QUE UBER NO PRESTA SERVICIOS DE TRANSPORTE O DE LOGÍSTICA O FUNCIONA COMO UNA EMPRESA DE TRANSPORTES Y QUE DICHOS SERVICIOS DE TRANSPORTE O LOGÍSTICA SE PRESTAN POR TERCEROS CONTRATISTAS INDEPENDIENTES, QUE NO ESTÁN EMPLEADOS POR UBER NI POR NINGUNA DE SUS AFILIADAS.

Conminando al usuario cliente a aceptar que no es una empresa de transporte y no tiene relación con el prestador transportista, ni UBER ni sus filiales.

Siendo más explícito en el punto 5 (Renuncias; limitaciones de responsabilidad; indemnidad) que prescribe asi:

RENUNCIA.

LOS SERVICIOS SE PROPORCIONAN “TAL CUAL" Y “COMO DISPONIBLES”. UBER RENUNCIA A TODA DECLARACIÓN Y GARANTÍA, EXPRESA, IMPLÍCITA O ESTATUTARIA, NO EXPRESAMENTE ESTABLECIDA EN ESTAS CONDICIONES, INCLUIDAS LAS GARANTÍAS IMPLÍCITAS DE COMERCIABILIDAD, IDONEIDAD PARA UN FIN PARTICULAR Y NO VIOLACIÓN. ADEMÁS, UBER NO HACE DECLARACIÓN NI PRESTA GARANTÍA ALGUNA RELATIVA A LA FIABILIDAD, PUNTUALIDAD, CALIDAD, IDONEIDAD O DISPONIBILIDAD DE LOS SERVICIOS O CUALQUIERA DE LOS SERVICIOS O BIENES SOLICITADOS A TRAVÉS DEL USO DE LOS SERVICIOS, O QUE LOS SERVICIOS NO SERÁN INTERRUMPIDOS O ESTARÁN LIBRES DE ERRORES. UBER NO GARANTIZA LA CALIDAD, IDONEIDAD, SEGURIDAD O HABILIDAD DE LOS TERCEROS PROVEEDORES. USTED ACUERDA QUE TODO RIESGO DERIVADO DE SU USO DE LOS SERVICIOS Y CUALQUIER SERVICIO O BIEN SOLICITADO EN RELACIÓN CON AQUELLOS SERÁ ÚNICAMENTE SUYO, EN LA MÁXIMA MEDIDA PERMITIDA POR LA LEY APLICABLE.

Uber renuncia a toda garantía y no es responsable de calidad, idoneidad, seguridad y el riesgo lo traslada a terceros proveedores.

LIMITACIÓN DE RESPONSABILIDAD.

UBER NO SERÁ RESPONSABLE DE DAÑOS INDIRECTOS, INCIDENTALES, ESPECIALES, EJEMPLARES, PUNITIVOS O EMERGENTES, INCLUIDOS EL LUCRO CESANTE, LA PÉRDIDA DE DATOS, LA LESIÓN PERSONAL O EL DAÑO A LA PROPIEDAD, NI DE PERJUICIOS RELATIVOS, O EN RELACIÓN CON, O DE OTRO MODO DERIVADOS DE CUALQUIER USO DE LOS SERVICIOS, INCLUSO AUNQUE UBER HAYA SIDO ADVERTIDO DE LA POSIBILIDAD DE DICHOS DAÑOS. UBER NO SERÁ RESPONSABLE DE CUALQUIER DAÑO, RESPONSABILIDAD O PÉRDIDA QUE DERIVEN DE: (I) SU USO O DEPENDENCIA DE LOS SERVICIOS O SU INCAPACIDAD PARA ACCEDER O UTILIZAR LOS SERVICIOS; O (ii) CUALQUIER TRANSACCIÓN O RELACIÓN ENTRE USTED Y CUALQUIER TERCERO PROVEEDOR, AUNQUE UBER HUBIERA SIDO ADVERTIDO 
DE LA POSIBILIDAD DE DICHOS DAÑOS. UBER NO SERÁ RESPONSABLE DEL RETRASO O DE LA FALTA DE EJECUCIÓN RESULTANTE DE CAUSAS QUE VAYAN MÁS ALLÁ DEL CONTROL RAZONABLE DE UBER. USTED RECONOCE QUE LOS TERCEROS PROVEEDORES DE TRANSPORTE QUE PRESTEN SERVICIOS DE TRANSPORTE SOLICITADOS A TRAVÉS DE ALGUNAS MARCAS DE PEDIDOS PODRÁN OFRECER SERVICIOS DE COCHE COMPARTIDO O ENTRE PARES (PEER-TO-PEER) Y PUEDE QUE NO DISPONGAN DE LA LICENCIA O DEL PERMISO PROFESIONAL. EN NINGÚN CASO LA RESPONSABILIDAD TOTAL DE UBER HACIA USTED EN RELACIÓN CON LOS SERVICIOS POR TODOS LOS DAÑOS, LAS PÉRDIDAS Y LOS JUICIOS PODRÁ EXCEDER DE QUINIENTOS EUROS (€500).

USTED PODRÁ UTILIZAR LOS SERVICIOS DE UBER PARA SOLICITAR Y PLANIFICAR SERVICIOS DE TRANSPORTE, BIENES O LOGÍSTICOS CON TERCEROS PROVEEDORES, PERO ACEPTA QUE UBER NO TIENE RESPONSABILIDAD ALGUNA HACIA USTED EN RELACIÓN CON CUALQUIER SERVICIO DE TRANSPORTE, BIENES O LOGÍSTICO PRESTADO A USTED POR TERCEROS PROVEEDORES QUE NO SEA COMO SE HA ESTABLECIDO EXPRESAMENTE EN ESTAS CONDICIONES.

LAS LIMITACIONES Y LA RENUNCIA EN ESTE APARTADO 5 NO PRETENDEN LIMITAR LA RESPONSABILIDAD O ALTERAR SUS DERECHOS COMO CONSUMIDOR QUE NO PUEDAN EXCLUIRSE SEGÚN LA LEY APLICABLE.

Agrega limitaciones de su responsabilidad tratando de exonerarse de cualquier vestigio de responsabilidad que de acuerdo a la equidad contractual, ello no debería corresponder; aunque no podemos negar que alude un monto exiguo de reconocimiento de todo daño: 500 dólares. La última parte de este acápite, como una salida para no negar derechos al consumidor anuncia que no pretende excluir de estos derechos al cliente o consumidor, sin embargo cuando analizamos las condiciones que prescribe el contrato observamos que son bastante restrictivas y causan incongruencias con la legalidad peruana, ya que de acuerdo al artículo 1398 para estos contratos de adhesión utilizados por Uber: “... no son válidas las estipulaciones que establezcan, en favor de quien las haya redactado, exoneraciones y limitaciones de responsabilidad...".

INDEMNIDAD.

USTED ACUERDA MANTENER INDEMNES Y RESPONDER FRENTE A UBER Y SUS CONSEJEROS, DIRECTORES, EMPLEADOS Y AGENTES POR CUALQUIER RECLAMACIÓN, DEMANDA, PÉRDIDA, RESPONSABILIDAD Y GASTO (INCLUIDOS LOS HONORARIOS DE ABOGADOS) QUE DERIVEN DE: (I) SU USO DE LOS SERVICIOS O SERVICIOS O BIENES OBTENIDOS A TRAVÉS DE SU USO DE LOS SERVICIOS; (II) SU INCUMPLIMIENTO O VIOLACIÓN DE CUALQUIERA DE ESTAS CONDICIONES; (III) EL USO POR PARTE DE UBER DE SU CONTENIDO DE USUARIO; O (IV) SU INFRAC- 
CIÓN DE LOS DERECHOS DE CUALQUIER TERCERO, INCLUIDOS TERCEROS PROVEEDORES.

Declara ser indemne a lo que haga el proveedor o el trasportista usuario, el que tiene que soportar reclamos, demandas, denuncias y gastos.

Con estas condiciones contractuales, esta empresa se enriquece indebidamente pues obvia el pago que por equidad le compete,ya que si alguien obtiene utilidad del trabajo de una persona y o el uso de un bien, resulta justo y razonable que tenga que responder con los riesgos que se origina como consecuencia deello ${ }^{3}$; interpretando el contrato, se coliga que esta empresa no asume riesgos, dejando el problema al transportista, que es un simple conductor que ha financiado a plazos su automóvil o bien está pagando un arriendo, siendo en su mayoría personas sin capacidad de asumir económicamente riesgos de accidentes, salvo el seguro público de accidentes SOAT. Pero lo más grave concurre cuando el mismo conductor ("un mal conductor") es contratado -sin mayor investigación personalpor Uber; y es el actor negativo de estos sucesos, es decir, el delincuente, que roba o hace tocamientos indebidos, viola y o quita la vida,entre otras actos delincuenciales, que desde luego son sucesos que atentan contra la dignidad humana y degradan la relación de consumo-servicio, pareciendo que estamos ante una situación de informalidad o anomia empresarial que no respeta la dignidad de la persona; pues como expresa Ramírez (2015, 43) si bien "la dignidad y la autonomía privada justifican que las personas puedan definir sus vidas a través del contrato, pero al mismo tiempo da un sustento necesario para advertir que no todos los contratos son dignos para la personas...";

Desde otro ángulo los términos del contrato que suscribe esta empresa con los conductores y usuarios para la prestación de servicios, son en la práctica impuestos unilateralmente por Uber, que trata de sobrepasar la normativa legal y aun constitucional peruana, aludido en este último caso, al artículo 1 referente a "la defensa humana de la persona humana y el respeto a su dignidad son el fin supremo de la sociedad y el Estado" Más aún, cuando esta empresa (como otras similares) desarman el concepto de tratadistas de gestión como Porter, quien da a entender

\footnotetext{
3 La teoría de caso fortuito parte de la premisa que si alguien obtiene utilidad de una persona o un bien , resulta justo y razonable que tenga que responder por los riesgos que se originen como consecuencia del uso de esta persona o bien. Mencionado en la casación No. CAS LAB No. 4258-2016 LIMA
} 
que lo más importante de una empresa es contribuir a una economía prospera de la comunidad o sociedad y no ser una de esas empresas que"solo buscan beneficios a corto plazo de forma engañosa o eludir consecuencias sociales...” (2009, p. 573); es por eso, añade Ramírez, en su tesis de Licenciatura 2015; que se hace perentorio la regulación estatal ya sea mejorando controles administrativos o aplicando la legislación de contratos, pues "...la intervención del Estado en el contrato de consumo estaría justificado en diferentes principios constitucionales, tales como el deber de velar por la dignidad humana..." (p.31); más aún si cae en cuenta que en la parte general de los contratos, en el artículo 1354 del CC se indica "que las partes pueden determinar libremente el contenido del contrato, siempre que no sean contrarios a la norma legal de carácter imperativo"; y en el caso específico del contrato de adhesión que suscribe Uber con sus usuarios, este contravendría la normas de orden público contenido en el artículo 1398 y que fuera detallado en un punto anterior; por lo tanto dicho contrato sería nulo e inaplicable conforme la legislación contractual peruana.

Siendo así, es necesario en el caso peruano repensar esta relación de consumo y recomponerla desde la óptica de un trato digno al consumidor, ya que clasificado como empresa de taxi le correspondería asumir responsabilidades en cuanto a incidentes, accidentes o actos ilícitos del conductor desde el inicio del servicio hasta el tramo final o llegada, y no solo ser un simple dador del acceso digital, de ahí la importancia de la Resolución del Tribunal contenida en el documento ECLI:EU:C:2017: 981, que incidiría en los demás países como el Perú, donde los derecho del consumidor es protegido a nivel constitucional:artículo 65 de nuestra constitución, como un derecho fundamental. Caso contrario se estaría dejando desprotegido a este consumidor, y desde luego, atentando contra la dignidad humana, tal como creemos estaría ocurriendo en nuestro país a las personas que han sufrido este tipo de situaciones ilícitas atentatorias contra su vida y o integridad física y psíquica. El cuadro No. 1 que presentamos, resume utilizando la triangulación de fuentes la relación de cómo piensan los consumidores sobre este transporte y la actuación de Uber ante los sucesos negativos acontecidos.

Cuadro No. 1. Resumen de triangulación de datos de encuestas y difusión de reportes periodístico por internet 
Pensamiento Crítico Vol. 23. $\mathrm{N}^{\circ} 1$

\section{(S.: Calificación de los encuestados sobre inseguridad, riesgos y necesidad de controles en este servicio de taxis).}

Respuestas de encuesta

1.- Que deben poner más control sobre el taxista.

2.- A veces cobran caro.

3.-Las empresas tienen q seguir con GPS a cada taxista y obligar a usar una línea radio para cada monitoreo.

4.-Es una buena opción para tomar un taxi ya q buscamos seguridad y buen trato. Pero a su vez hav q tener cuidado para no correr ningún riesgo.

5.-No confío en ningún taxi...Sólo tomó colectivos donde van varias personas y tiene sus panaderos ya definidos

6.-Las medidas legales y regulatorias aún no han avanzado suficiente para tomar control de dichos sucesos.

7.-Esas empresas no están reguladas por el estado y hacen lo q les da la gana. Sólo hav un $50 \%$ de confianze.

8.-Que ese tipo de personad son dañinos para esta sociedad y que lamentablemente nadie esta a salvo de ellos.

11.- no es seguro este tipo de servicios.

12.-Son cosas que se pueden superar, talvez

mejorando la aplicación.

13.- Trafico.

14.- Pienso que las empresas encargadas de ofrecer este servicio deberían tener más contro sobre el personal que contratan a su servicio

15.- Que debe haber mejor filtro al escoger a los conductores.

16.- Hasta ahora no he presenciado ninguno.

17.- Cualquier tipo de servicio/empresa tiene riesgo de sufrir estos incidentes; más aún, as el servicio de taxi online mejora muchísimo la experiencia de viajar a un bajo costo, rápido y más seguro.

18.-Debería de haber más control en la contratación del personal en este caso los choferes. Verificando su historial, haciendo pruebas psicológicas, etc.

19.-Bueno

20.- Que no todo siempre resulta ser tan bueno ya que hay conductores que les falta conocer sobre educaciónvial.

21.- Bueno en todo servicio actualmente no es nada seguro pero este servicio a pesar de aquellos acontecimientos ofrecen un servicio más seguro que tomar un taxi cualquiera de la calle.
Casos

Consideraciones
X CASO 1.- "Sin embargo, el presunto taxista desvió su ruta y le robó todas sus pertenencias. Lo más indignante es que, según

X afirmó, la empresa no ha querido ayudarla. conladenuncia."http://larepublica.pe/ sociedad/1105891-pasajera-denuncia-que-fueasaltada-por-taxista-de-aplicativo-uber-video.

X

CASO 2.- "...ella se dio cuenta que el chofer cambió deruta lo que la puso en alerta, además de sentír que perdía la movilidad del cuerpo, la capacidad de hablar y a sentir sueño. Gracia

X Farje explicó que sabía que el conductor del servicio de Uber la había drogado. Desactivaron el conductor https://diariocorreo.pe/ciudad/ facebook-joven-denuncia-que-chofer-de-uber-

X la-drogo-en-surquillo-fotos-700970/

Caso 3y 4:Uber:Dosusuariasy dosterribles experiencias con elmismo conductor.Una de las jóvenes denunció un intento de secuestro la otra ni siquiera tomó el servicio, pero fue víctimade acoso. Los representantes de la empresa, mediante un correo electrónico, "Le comunicaron que habían tomado las medidas necesarias." http://larepublica.pe/ sociedad/878720-uber-dos-usuarias-y-dosterribles-experiencias-con-el-mismo-conductor. 24 Mayo 2017| 8:54

Caso 5 :Uber:sentenciana 19añosdeprisióna choferqueviolóa pasajera $\mathrm{htt}$ ://larepublica. pe/mundo/876979-uber-sentencia-19-anosde-prision-chofer-que-violo-pasajera $18 \mathrm{de}$ mayo 2017

Otras fuentes en internet

-Lo que más me interesaba saber era qué pasaría conmigo, el pasajero, si es que sufriera un robo o si es que el taxista decidiera sacar una Colt de entre sus piernas. ¿Qué me ofrecen estas empresas en cuanto a seguridad?http://curwen utero pe/2016/09/12/los-taxis-segurospor-aplicacion-no-son-tan-seguros-comoimaginabas/

- ¿Uber no se hace responsable? ¿Cómo puede decir que nos ofrece un transporte seguro sialfin y al cabo es solo un reclutador de choferes Como ella misma dice en el correo enviado? tendrian que haberla herido y golpeado o violentado de maneras inimaginables para que recién pueda recibir algún tipo de respuesta? ¿Qué necesita la transnacional Uber Taxi para atender a alguien que denuncia haber sido atacada en una de sus unidades? ¿Qué entidad del Estado regula este tipo de servicios? http:// utero.pe/2016/03/20/la-respuesta-de-uberdel-taxista-y-de-la-denunciante-despues-delincidente/
De la triangulación aplicada entre las respuestas de la pregunta abierta y los casos periodísticos y o de interne (señaladas en negrita, cursivas y subrayado para identificar lo triangulado), convergemosque en realidad en el mercado se han presentado diversas sucesos negativos como asaltos, acosos, intentos de violación o violación que atentan con la dignidad humana desdiciendo desde una óptica empresarial la seguridad, confiablidad y calidad del servicio al menos en algunos casos, lo cual si bien son pocos, demuestran un maltrato la usuario pasajero que corrobora con la opinión de 17 de los participantes que indican que siempre asumen riesgos en la seguridad al tomar estos taxis por lo que asumen mejoras en el control en el conductor para tener mayor seguridad.

Lo que hace UBER ante una denuncia es desactivar al chofer "temporalmente" e investigar hasta lo que le conviene ya que el contrato lo hace inmune a tod responsabilidad.

Creemos de acuerdo a nuestra legislación el Estado le corresponde la tutela del consumidor y asumir algun regulación para mejorar el trato de conformidad con la ley y la constitución empero sin restringir el avance innovación tecnológica. Más del otro lado tampoco a nombre de la tecnología, su innovación y el mercado se pued atentar contra la dignidad humana.

Fuente: propia 


\section{El código de consumo y el servicio de APP de taxis (UBER) en relación al consumo digno en el Perú.}

Tal como se refiere en líneas anteriores, la dignidad humana es un principio elemental del derecho natural que le asiste a toda persona humana de ser respetado en sus derechos empezando desde los más elementales como la vida, la salud y la integridad física psíquica. Ello supone que en una relación especifica de consumo, el consumidor en tanto ser humano, le corresponde un trato adecuado a su dignidad humana, es decir un trato digno. Y si la legislación no lo ha establecido en forma literal, ello no es óbice para no responsabilizar y dispensar al proveedor del sistema: Uber, por el trato indigno o deshonroso al consumidor peruano,cuando ocurre un evento dañoso (atentado contra la vida, salud, integridad física, libertad e inclusive daño a la propiedad), y dejando al "eventual" transportista o conductor de taxi(usuario conductor según Uber),la responsabilidad de toda sucesoque afecte al cliente consumidor; más aún cuando este lucra por dicho servicio y como tal por equidad le corresponde las obligaciones derivadas de este negocio.

Por otro lado, apuntando al artículo 65 de la constitución, este prescribe que "El estado defiende el interés de los consumidores y usuarios (...) en el mercado. Asimismo vela, por la salud y la seguridad de la población. Observándose en el último párrafo, el derecho de todo ciudadano como miembro de la población que el Estado tutele la seguridad del ciudadano cliente en la prestación de este servicio de taxis. Más esto no se agota con la norma constitucional, pues normas de menor jerarquía, como el código de consumo (Ley No. 29571) contiene ciertos principios aplicables y correlativos a cualquier protección que pudiera dejarse de lado; así el principio pro consumidor refiere que en caso de duda insalvable en los contratos se debe interpretar en el sentido más favorable al consumidor.

Más aun las políticas que señala estecódigo, concuerdan con el ordenamiento jurídico en pos de su tutela, puesto que según Latorre “... existe una estrecha relación entre las nomas publicas impartidas en el ordenamiento jurídico que regula la economía y las políticas públicas destinadas a la protección de los consumidores", Latorre et al. (2016a, p. 1-12). En tal sentido se detalla las concordancias de alguna de estas políticas y la articulación correspondiente que conjugan en función en pos de la defensa de la persona en tanto ciudadano consumidor, a saber: 
1.-El Estado protege la salud y seguridad de los consumidores a través de una normativa...y fiscaliza su cumplimiento a través de los organismos competentes.

8.-El Estado procura y promueve una cultura de protección al consumidor y comportamiento de acuerdo a la buena fe de los proveedores...para asegurar el respeto y pleno ejercicio de los derechos reconocidos en el presente código..."

11.-El Estado orienta sus acciones para que la protección al consumidor sea una política transversal que involucre a todos los poderes públicos, así como la sociedad. (...)

En comparsa a lo anterior, el artículo 5, inciso 1. 1 (a) indica el: "Derecho a la protección eficaz respecto de los productos y servicios que, en condiciones normales o previsibles, representen riesgo o peligro para la vida, salud e integridad física.; (i) Derecho a la reparación y a la indemnización por daños y perjuicios...",entre otros derechos.

Asimismo al proveedor de acuerdo con el artículo 104, es administrativamente responsable por la falta de idoneidad o calidad, el riesgo injustificado...sobre un producto o servicio determinado", sin prejuicio de las responsabilidades civiles y penales que correspondan.

En tal sentido el código del consumidor contempla la tutela del consumidor y la regulación del Estado, aunque reagrupado en condiciones normativas más específicas a este tipo de servicios de aplicaciones digitales, pues la realidad así lo exige, y no que bajo elparaguas de una economía de nuevo cuño, según Latorre et al. (2016a, pp. 1-12), "surgida de una sociedad contemporánea interrelacionado en sistema globalizado”,se pueda actuar con libertinaje y se permita cualquier abuso; más aun, tratándose de la integridad psíquica física y la vida de las personas. $\mathrm{Ni}$ tampoco con ello se pretende detener la dinámica de toda sociedad de avanzar y renovarse.

Es por ello qué, consumo digno, significa en la prestación de servicios de transporte de los taxis con app, la obligación como sistema empresarial de garantizar la seguridad del usuario o consumidor, es decir satisfacer su consumo con un trato digno en todo el tramo del servicio (el contrato de transporte es de resultado), que se traduce en la seguridad y bienestar del consumidor, ya que este espera un entorno confiable y seguro en la consecución de su consumo; ello no significa, que si bien se tiene un alto porcentaje de delincuencia y una seguridad pública deficiente, se le exija al prestador de estos servicios mejorar la seguridad pública, que es atinente 
al sector estatal; si no, solo suministrar la seguridad de su consumidor dentro del servicio propio; sin embargo si pese a ello sucediera algún incidente, tendrá la responsabilidad de afrontar, desde luego previa investigación, lo que le corresponde según los hechos y el derecho, y no eludir su responsabilidad.

No se trata de ningún manera detener la comercialización y prestación de un novedoso servicio puestos al servicio de la sociedad, si no que este tipo de empresas, así como se benefician y obtienen ganancias, no se responsabilice de la ocurrencia de riesgos que pueda sufrir el clienteconsumidor como producto de la prestación de este servicio, y en consecuencia,se lucre a costa de otros (usuario conductor) que no gozan de la mejor opción rentista que si la recibe la empresa transnacional Uber, ni tampoco estaría en condiciones de pagar las indemnizaciones pertinentes o solventar los daños que sufra el cliente consumidor; produciéndose así diversas injusticias, sin que las instituciones públicas muestren decisiones eficientes y efectivas para equilibrar estas relaciones que engendran desafíos en estos tiempos de hiper-consumo, macdonalización de la sociedad y virtualización de los negocios, Latorre et al. (2016b, pp. 175-188). Más aun, esto es más grave cuando las instituciones públicas suele ignorar estas situaciones a costa de obnubilarse por los sistemas comerciales modernos del sector privado; siendo más, cuando son consideradas por estas, como el ideal de la optimización, más aun, alzaprimando con inclinación triunfante la opción privatista sobre lo público, optando muchas veces por acoplarse a esta modernidad, sin mayores reparos a su rol regulador y ordenador de la sociedad, según Stiglitz obviando que "un cambio en la tecnología o en la estructura del mercado ...inevitablemente va acompañado de unos cambios sociales de un enorme magnitud, incluyendo ideas sobre cómo debería organizarse la sociedad y la economía" Stiglitz (2012, p.181); dando lugar que los privados se sientan con aparentes derechos de liderar todo tipo de negocios y desde luego sobrepasar derechos de los consumidores, pues esto va de la mano con lo que expresa Latorre, quien sintetiza que "el derecho vive, hoy por hoy, un acople entre el gerencialismo desmedido y el éxito de los resultados, contrastable con la precaria y cada vez más limitada satisfacción colectiva de los derechos esenciales del ser humano" Latorre (2016b, pp. 175-188). 
Por lo mismo se debe tener en cuenta que el Estado y por ende sus instituciones,que esta problemática en apariencia micro económica o de mercado, también se ven involucrado otros sectores sociales (macroeconomía), a tenor de lo que expresa el economista Alarco ${ }^{4}$

Desafortunadamente, todos los servicios con base al uso de internet conducen a una reducción de los ingresos del Estado en perjuicio de todos. Estas es una preocupación a nivel internacional. La proliferación de estos reduce la presión tributaria en circunstancias de las necesidades y costos de los gobiernos que crecen. De donde van a salir los recursos para los servicios públicos tradicionales, inversión pública y función retributiva del Estado? (...) En realidad el mundo de los Airbnb, Uber y similares elevan la informalidad y precarizan a la sociedad. Efectivamente, liberan ingresos a los consumidores medios para otros usos, pero nada garantiza que en el mundo real se reciban mayores servicios y se beneficie o todos.

En tal sentido es loables que nuestros sectores públicos revitalicen sus funciones que le competen por ley, no en el sentido de nadar contra la corriente, impidiendo o limitando la modernidad, si no asumir la función que le corresponde de acuerdo al contrato social que se refleja y esta prescribe el artículo 1 de nuestra constitución y así como el artículo 65 del mismo cuerpo legal, ya que en toda sociedad futura o sociedad donde está consolidando un nueva realidad -como creemos es esta forma de hacer negocios de taxis en la denominada economía colaborativa-, "...su característica fundamental, como han sido la anterior, involucra nuevas instituciones y problemas"(Drucker 2005, p. 247), al que le agregaríamos nueva políticas, y posibles regulaciones, en el afán de no desproteger al ser humano en cualquier opción actual o futurista de la sociedad.

\section{Conclusiones}

- La existencia de estas empresas y otras similares, en los países es evidente. La innovación en la producción y comercialización de estos servicios a través de combinación de sistemas digitales y físicos desagregados outsourcinghan impactado a las normas regulatorias que la administración pública tiene como clásicas,

4 Contestación al Abogado Alfredo Bullard, defensor del neoliberalismo, por su artículo "Monopolio solapa" (El comercio 31 de marzo del 2018), en sus compañas de desregulación económica desde nuestro de punta de vista muy sesgada a las grande ventajas de la desregulación pero sin abordar o ignorar y o contrarrestar otras consecuencias que son reales a la otros grupos de la sociedad. 
originándose distorsiones de mercado y propiciando algunas veces informalidad.

- Si bien la dinámica tecnológica, social y cultural preludia una modernidad más cómoda para la vida humana, ello no es óbice para que este progreso nuble nuestra sensibilidad de que somos seres humanos y por lo tanto nos corresponde un trato justo en cualquier tiempo o posición que ocupemos en la sociedad y el mercado.

- En la denominada economía colaborativa, se enerva con mayor impulso, las relaciones jurídicas empresa estado, empresa trabajador, empresa mercado, entre otras que deben ajustarse a la luz de la equidad y el derecho justo.

- El Estado, debe evaluar diversas fisuras que geste situaciones inequitativas para tener argumentos y el instrumento para ordenar a esta empresa y similares en el mercado, pero insistimos que no sólo es de regulación o plasmación de leyes, si no controles y supervisión eficaz.

- La clásica industria de taxis tendrá que tomar las medidas más convenientes según corresponda el escenario en cada país, y considerar esta problemática como un desafío, que nos está dando la oportunidad de salir de tranquilidad empresarial, que nos fuerza a pensar y actuar como enfrentar a esta nueva competencia y otras que se presenten en el futuro

- A nivel latinoamericano debemos coordinar los esfuerzos comunes para dar soluciones concordantes a un problema común que afecta a los países.

\section{Referencias bibliográficas}

Armas, C. (2016). El negocio de las apps de taxis y la economía colaborativa: ¿relaciones no laborales o laborales, según los términos contractuales que lo sustentan (caso perú)?.En Neuman Business Review, vol 2 - num. 2 Escuela de Pos grado Neuman Business Review, Tacna, pp.9-25.

Alarco, G. (2018). Impactos económicos de Airbnb y Uber, El Dia, Sección economía. Lima, 06 de abril del 2018. 
Bullard, A. (2018). Monopolio solapa. Lima. El Comercio. Lima, 31 de marzo del 2018.

Cabrera, K. (2011). Desde la teoría clásica del contrato hasta los nuevos contratos. En: Revista de Derecho No. 35 Barranquilla, p.55-95.

Corte Suprema de Justicia del Perú(2017). Casación Laboral No. 4258-2016 Lima publicado en Peruano el 30 de enero del 2017, p.87130.

Diez, S. (2015). La economía colaborativa: un nuevo modelo de consumo que requiere la atención de la política económica. Universidad de Valladolid, p. 8

Dinero (2015) Magnates de las apps. Revista digital. Encontrada el 12 de marzo del 2017 en hppt://www.dinero.com/edicion-impresa/caratula/articulo/ aplicaciones-mas-usadas-mundo-2015/208936)

Drucker, P. (2005). La gerencia en la sociedad futura. Madrid: Buena Semilla. p.250.

Elías, M. (2017). Congreso de la República del Perú. Proyecto de Ley No. 1505/2016-CR de 08 de Junio del 2017, p.12.

Grupo de alumnos curso derecho económico 2016-N ciclo 2017-II, encuesta realizada por internet.

Flick, U. (2014). La gestión de la calidad en investigación cualitativa. Madrid: Ediciones Morata.P.67.

De Haro, G. y Cereijo, M. (2016). Los límites de la economía colaborativa. OBS Business School Universidad de Barcelona, España, p.36. Encontrado en www.obs.edu.pe

El Correo (2017). Joven denunció que el conductor cambió repentinamente de ruta durante el viaje. Encontrado el 03 de marzo del 2017 en: https:// diariocorreo.pe/ciudad/facebook-joven-denuncia-que-chofer-de-uberla-drogo-en-surquillo-fotos-700970/27 de Septiembre del 2016 - 20:10» Textos: Redacción Multimedia » Fotos: Facebook.

Lago, S. (2016). Economía Colaborativa: ¿Pondrá fin a los negocios tradicionales? Entrevista enRevista actual IT No. 9, KIBERNUM Chile, pp. 16-20.

Lara, R. (2016). Proyecto de Ley SN difundido por el Capitolio Nacional, p.17 ok.

Latorre, E. y Rodríguez, A. (2016a). Los derechos del consumidor en Colombia y su constitucionalización desde el Enfoque de los derechos sociales. En Ponencia denominada "Programa Consuma Caribe que gano el proceso de convocatoria de Colciencias" Grupo de Investigación Escuela de Derecho de la Universidad Sergio Arboleda Seccional Santa María. Colombia p. 1-12. 
Latorre E., Restrepo, J. y Gómez, M. (2016b). La necesidad de repensar el derecho. Hiperconsumo y macdonalización de la sociedad. En: ADVOCATUS, volumen 14 No. 27 Universidad Libre seccional Barranquilla, p.175-188.

Martínez, V. (2013). Reflexiones sobre la dignidad humana en la actualidad Bol. Der Comp. vol.46 no.136 México ene/abr. p.1-18. Encontrado el 20 de marzo en versión On-line ISSN 2448-4873versión impresa ISSN 0041-8633

Porter, M. (2009). Ser Competitivo Tomo II. Ediciones Deusto: Barcelona, España, $623 p$.

Ramírez, V. (2015). Derecho del Consumo. Un breve relato sobre sus fundamentos teóricos, desarrollos y nuevas tendencias. Tesis para optar el grado de Licenciado en ciencias jurídicas y Sociales, Universidad de Chile, Chile, p.17.

La República (2017) Pasajera denuncia que fue asaltada por taxista de aplicativo Uber [VIDEO] 3 Oct 2017, Encontrado el 03 de marzo del 2018 http://larepublica. pe/sociedad/1105891-pasajera-denuncia-que-fue-asaltada-por-taxista-deaplicativo-uber-video

La República (2017) Dos terribles experiencias con el mismo conductor. Encontrado en Marzo del 2018 http://larepublica.pe/sociedad/878720uber-dos-usuarias-y-dos-terribles-experiencias-con-el-mismo-conductor. 24 Mayo 2017 | 8:54 h

La República. Chofer que violo a pasajera sentenciado a 19 años de prisión. Encontrado el 3 de marzo 2018 en: http://larepublica.pe/mundo/876979uber-sentencia-19-anos-de-prision-chofer-que-violo-pasajera 18 de mayo 2017.

Real Academia Española (2014). Diccionario de la Real Academia Española de la Lengua. Madrid: Vigésima tercera edición. Edición tricentenario Espasa, p. 799.

Tribunal de Justicia de Luxemburgo Sentencia (2017). Resolución ECLI:EU:C:2917:981 de la Sentencia del Tribunal de Justicia (Gran Sala) del 20 de diciembre de 2107 Asunto C-434/15, en el procedimiento entre Asociación Profesional Elite Taxi Y UberSystemsSpain, S. L.

Sholz, T. (2016) Cooperativismo de Plataforma: Desafiando la economía corporativa. Digital CommonsResarchGroupUniversitatOberta de Catalunya, p. 7.

Stiglitz, J. (2012). El precio de la desigualdad. Santillana. Madrid: Ediciones Generales.S. L., p.415 . 
Ventura, R. (2017). Congreso de la República del Perú. Proyecto de Ley No. 2218/2017-CR de 06 de diciembre del 2017, p.7 .

Uber (2017). Términos y condiciones encontrado el 04 de diciembre del 2017 en : https://www.uber.com/es-PE/legal/terms/pe/

Utero.pe (2017). Blog Indignante: pasajera denuncia haber sido atacada en un taxi de la transnacional Uber y en la empresa no le responden. Encontrado en diciembre de 2107 http://utero.pe/2016/03/20/la-respuesta-de-uberdel-taxista-y-de-la-denunciante-despues-del-incidente/

Utero.pe http://curwen.utero.pe/2016/09/12/los-taxis-seguros-por-aplicacionno-son-tan-seguros-como-imaginabas/Encontrado en diciembre de 2017 
